\title{
Binarity in the Orion Trapezium Cluster
}

\author{
Mark J. McCaughrean \\ Astrophysikalisches Institut Potsdam, \\ An der Sternwarte 16, 14482 Potsdam, Germany
}

\begin{abstract}
We summarise the results of recent optical and near-infrared imaging studies of the binary fraction among young low-mass stars in the dense Orion Trapezium Cluster. Over the separation range $\sim 30-500 \mathrm{AU}$ and within the observational errors, there appears to be no excess of binary systems in the cluster relative to the main sequence field star population. Over the separation range $\sim 1000-5000 \mathrm{AU}$, the cluster is deficient in binaries relative to the field. Both results are in contrast to those found for the more distributed population of young stars in the Taurus-Auriga dark clouds, which is overabundant in binaries by roughly a factor of two. We briefly discuss possible origins for this difference and observational tests which may distinguish between them, and the implications these results have for our understanding of the typical environment where most young stars are born.
\end{abstract}

\section{Introduction}

The discovery of an overabundance of young low-mass binary systems in lowmass dark cloud star-forming regions, most notably Taurus-Auriga (see, e.g., Leinert etal. 1993; Ghez, Matthews, \& Neugebauer 1993; Köhler \& Leinert 1998; Duchêne 1999; and many papers in these proceedings) relative to those found on the main sequence in the field (e.g., Duquennoy \& Mayor 1991 [DM91] for field G-dwarfs) poses a thorny dilemma for our understanding of the star formation process. If almost every star in Taurus-Auriga forms in a binary or higher-order multiple system, how can this fraction be reduced to only $\sim 50 \%$ by the time these stars reach the field?

There are two possible solutions in the context of Taurus-Auriga itself. First, the distribution of binary semi-major axes in Taurus-Auriga may be much narrower than for the field and peaked within the $\sim 15-1500$ AU range typically observable with infrared speckle, adaptive optics, and direct imaging techniques at the $\sim 140 \mathrm{pc}$ distance to Taurus-Auriga. The overall frequency of binary systems might then still be consistent with the field if there were a dearth of binaries outside the 15-1500 AU range, with subsequent evolution of the orbital parameters broadening the distribution as the stars diffuse into the field. However, lunar occultation observations in Taurus-Auriga show binaries to be overabundant even at 1-3 AU (Richichi et al. 1994; Simon et al. 1995) and spectroscopic binary systems are not abnormally rare (Mathieu 1994), thus this solution seems unlikely. Second, perhaps dynamical interactions between young stars disrupt 
the binaries and lower the fraction of such systems. However, this hypothesis is unviable in Taurus-Auriga. At typical volume densities of $10 \mathrm{stars} \mathrm{pc}^{-3}$ (Gomez et al. 1993) and a 3D velocity dispersion of $\sim 1.5-3.5 \mathrm{~km} \mathrm{~s}^{-1}$ (Jones \& Herbig 1979), the timescale for interactions between pairs of binaries with a separation on the order of $1000 \mathrm{AU}$ is $\sim 5 \times 10^{8} \mathrm{yrs}$, too long to be effective before the stars dissolve into the field.

Fortunately, there is a more global hypothesis, namely that Taurus-Auriga is a cosmic red herring. Most star-forming material in the galaxy is found in giant molecular clouds (GMCs; $\sim 10^{4}-10^{6} \mathrm{M}_{\odot}$ ) and not low-mass dark clouds like the Taurus-Auriga complex $\left(\sim 10^{3} \mathrm{M}_{\odot}\right)$ (Combes 1991). In the GMCs, most stars form in dense clusters $\left(10^{3}-10^{5} \mathrm{stars}^{-3}\right)$ rather than in isolation or relatively small groups $\left(\sim 1-10\right.$ stars pc $\left.^{-3}\right)$ (Lada, Strom, \& Myers 1993; Zinnecker, McCaughrean, \& Wilking 1993; Meyer et al. 2000; Clarke et al. 2000). Thus, the birth and early evolution environment for most stars may be very different to that found in Taurus-Auriga, and we need to examine the binary population in those more typical regions, to see if it is more like that of the field.

\section{The Orion Trapezium Cluster}

Taurus-Auriga provides much of the basis of our present paradigm for star formation simply because it is one of the nearest star-forming regions to the Sun, and thus the most readily studied. The nearest young clusters are two to three times further away (IC 348 at $300 \mathrm{pc}$ and the Orion clusters at $450 \mathrm{pc}$ ), thus making it harder to detect and resolve stars, binaries, and disks in them.

The nearest (and thus prototypical) GMCs with ongoing vigorous star formation are Orion A and B (L 1641 and L 1630) at 400-500 pc (Tatematsu \& Wilson 2001; Launhardt \& Lada 2001). Within these clouds there are dense clusters associated with NGC 2024, NGC 2023, NGC 2071, L 1641-N, and OMC$2 / 3$, for example (Meyer \& Lada 2001; Allen \& Hillenbrand 2001), all crowned by the Orion Nebula or Trapezium Cluster (Herbig \& Terndrup 1986; Zinnecker et al. 1993; Hillenbrand 1997) in L 1641. The cluster contains 2000 members within a radius of $\sim 15$ arcmin or $\sim 2$ pc (Hillenbrand \& Hartmann 1998), with masses spanning the IMF from OB stars to brown dwarfs (McCaughrean et al. 1996; Hillenbrand \& Carpenter 2000; Luhman et al. 2000). In the core, 50 stars lie within a radius of $\sim 0.1 \mathrm{pc}$ of the eponymous Trapezium OB stars, yielding a density of $\sim 5 \times 10^{4}$ stars pc $^{-3}$ (McCaughrean \& Stauffer 1994). The cluster is also a rich hunting ground for associated phenomena such as ionised and silhouette circumstellar disks, microjets, and large-scale outflows (see, e.g., Bally, O'Dell, \& McCaughrean 2000; McCaughrean 2001).

As it is the largest of the Orion clusters, it is tempting to think of the Trapezium Cluster as an extreme, and that most stars probably form in lowerdensity regions. However, precisely because it is the largest cluster, it accounts for a large fraction $(\sim 50 \%)$ of all stars that have formed in the Orion A GMC within the last $\sim 1 \mathrm{Myr}$. Also, much more extreme (larger, denser) clusters are found elsewhere in the galaxy (e.g., NGC 3603 in Carina at $6.5 \mathrm{kpc}$; Brandl et al. 1999), but are less well-studied simply because they are further away. Thus, at least qualitatively, it is reasonable to consider the Trapezium Cluster as a typical star-forming environment for the present purposes. 


\section{Observational Constraints}

Surveys for binary systems in the Trapezium Cluster are harder than in TaurusAuriga. First, its $450 \mathrm{pc}$ distance means that similar age and mass stars will be ten times fainter in the Trapezium Cluster than in Taurus-Auriga, thus requiring deeper observations in order to make a direct comparison: in particular, the speckle technique used to great effect in Taurus-Auriga (Leinert et al. 1993; Ghez et al. 1993) runs out of sensitivity in the Trapezium Cluster and is much less useful at least for low-mass stars. Second, equal angular resolution observations yield only one third of the linear resolution in the Trapezium compared to Taurus-Auriga. Thus, for diffraction-limited observations with a 4-m class telescope at $2 \mu \mathrm{m}$, a resolution of $17 \mathrm{AU}$ is achievable in Taurus-Auriga, but only $55 \mathrm{AU}$ in the Trapezium Cluster. This difference is crucial, since the typical separation for low-mass field binaries lies in between, at $~ 30 \mathrm{AU}$ (DM91). Third, the much greater stellar density in the Trapezium Cluster makes it very hard to identify wide binaries. This outer limit is generally set by the separation at which confusion sets in, i.e., when the chance projection of another unrelated young or background field star comes becomes appreciable. In Taurus-Auriga this occurs at $\sim 30$ arcsec or $4200 \mathrm{AU}$ (see, e.g., Reipurth \& Zinnecker 1993), while in the core of the Trapezium Cluster it is at $\sim 1 \operatorname{arcsec}$ or 450 AU only. Fourth, the lunar occultation technique that has been used to search for small separation binaries in Taurus-Auriga is not applicable to the Trapezium Cluster, simply because it is never occulted by the Moon.

Thus overall, it is hard to obtain observations of the binary population in the Trapezium Cluster that are directly comparable to those made for TaurusAuriga, and in general the range of projected separations that can be probed is narrower. There is, however, one major compensation: the Trapezium Cluster contains so many members within a relatively small field that good statistics are in principle relatively easy to come by.

\section{Observational Techniques}

Despite the difficulties described above, the binary population of the Trapezium Cluster was studied extensively in the 1990s using a number of observational techniques. In this section, we outline these techniques and their various advantages and disadvantages, before reviewing and analysing the results.

- Direct imaging: The WF/PC and WFPC-2 cameras on the Hubble Space Telescope have been used to make near-diffraction-limited surveys of large areas of the cluster at optical wavelengths (Prosser et al. 1994; Padgett, Strom, \& Ghez 1995; Patten et al. 1999), with linear resolutions of $\sim 40 \mathrm{AU}$ at longer wavelengths $(\sim 0.8 \mu \mathrm{m})$ where many members of the partially embedded population can be detected against the bright background nebula. Some limited regions of the cluster, including more embedded stars, have also been imaged with NICMOS at $\sim 2 \mu \mathrm{m}$, with a resolution of $\sim 90 \mathrm{AU}$ (Stolovy et al. 1998).

- Speckle imaging: The bispectrum speckle masking technique has been used to observe the bright $\mathrm{OB}$ stars in the Trapezium Cluster at near-infrared wavelengths on the Russian $6 \mathrm{~m}$ telescope, with linear resolutions of $\sim 40 \mathrm{AU}$ 
(Weigelt etal. 1999; Preibisch etal. 1999; Preibisch, Weigelt, \& Zinnecker, these proceedings). However, this technique cannot probe to much lower masses at this distance due to sensitivity limitations.

- Speckle holography: This modified speckle technique relies on the presence of one or more bright stars within the field-of-view which can be used as a post-detection wavefront-correction or deconvolution key, which enables an enhanced sensitivity limit over the normal technique. Speckle holography has been used at near-infrared wavelengths in the core of the cluster where one of the bright Trapezium OB stars can be used as the reference source, with linear resolutions on the Calar Alto $3.5 \mathrm{~m}$ telescope of $\sim 65 \mathrm{AU}$ at $2.2 \mu \mathrm{m}$ (Petr et al. 1998; Petr 1998).

- Adaptive optics: This technique relies on bright stars within the field-ofview to allow real-time wavefront error correction and thus near-diffractionlimited spatial resolution combined with long, high-sensitivity imaging observations. Simon, Close, \& Beck (1998) made adaptive optics observations at near-infrared wavelengths on the University of Hawaii $2.2 \mathrm{~m}$ telescope to provide linear resolutions of $\sim 100 \mathrm{AU}$ over a substantial area in the cluster, while similar observations by Petr, McCaughrean, \& Zinnecker (2001; see also Petr 1998) using the ADONIS system on the larger ESO $3.6 \mathrm{~m}$ telescope yielded a better resolution of $\sim 65 \mathrm{AU}$ albeit over a more limited area surrounding a number of bright cluster members. Figure 1 shows a section of these data centred on the Trapezium OB stars for illustration purposes: more detail can be found in Petr (1998).

\section{Observational Results}

In this paper, we concentrate on the key results for the lower-mass stars, in order to make the most appropriate comparison with the field G-dwarf survey of DM91 and the various Taurus-Auriga surveys. The multiplicity of massive OB stars in the Trapezium Cluster is discussed in the paper by Preibisch et al. in these proceedings, where the surprising result is found that the multiplicity is extremely high, with each OB star having more than one companion on average. At face value, when compared with the results for the low-mass stars discussed here, it appears as though the binary systems with massive primaries must have formed via a different mechanism compared to their lower-mass cousins.

\subsection{Close Visual Binaries}

Table 1 summarises the results from the majority of the observational surveys listed in Section 4. In each case, the derived binary fraction over the given separation range is shown next to the corresponding fraction for the field star population over the same range from DM91, as described in the table caption. At first sight, the comparisons with the field are rather scattered, but a pattern emerges under somewhat closer scrutiny. In the surveys of Padgett et al. (1997) and Stolovy et al. (1998), the Trapezium Cluster binary frequency is higher than that of the field, but only by $\sim 1 \sigma$. This result may be real, insofar as the Padgett et al. survey covered the outer reaches of the cluster where (for example) 


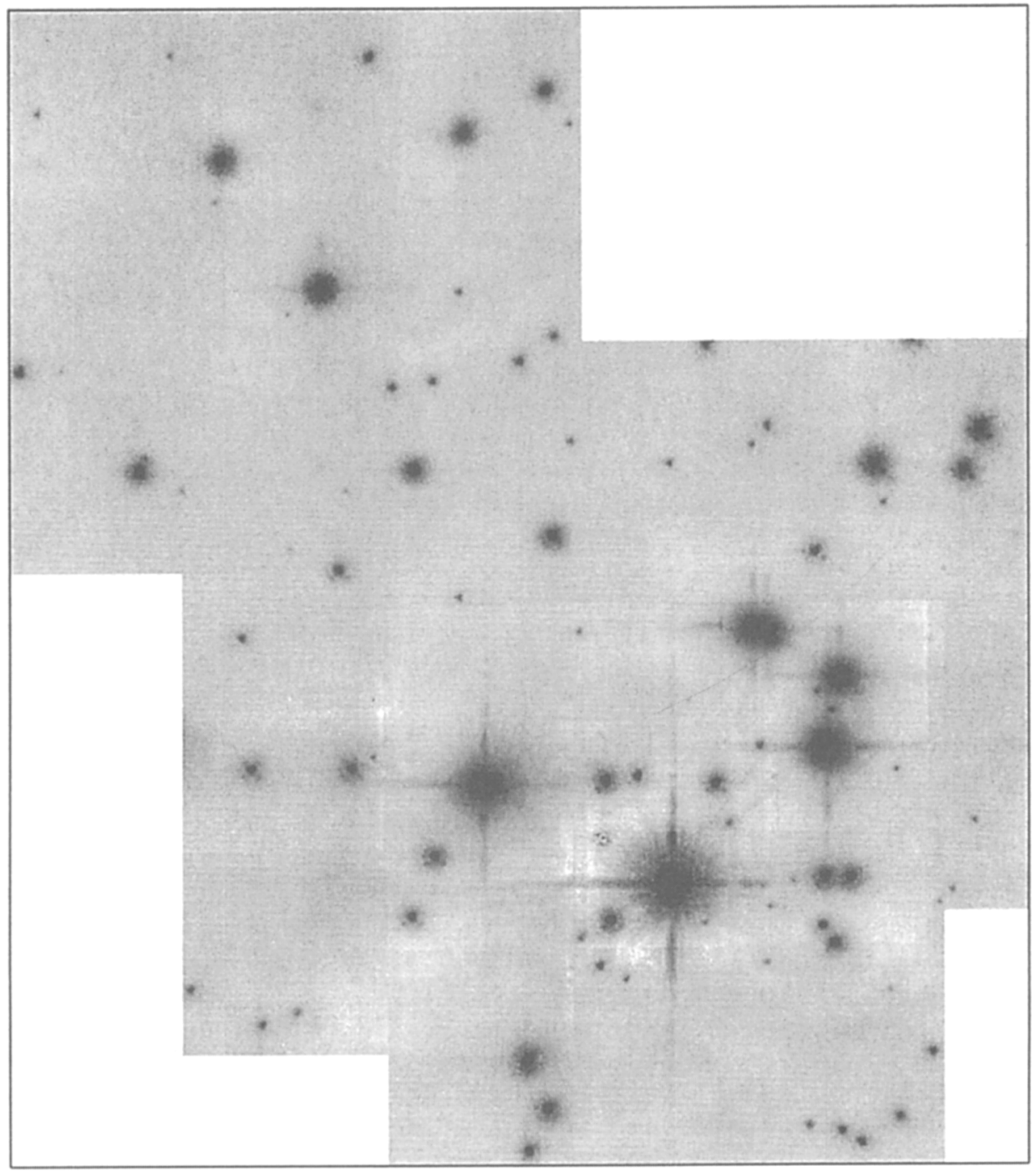

Figure 1. A section of the K-band adaptive optics survey of the Trapezium Cluster by Petr, McCaughrean, \& Zinnecker (2001; see also Petr 1998), made with the ADONIS AO system on the ESO 3.6-m telescope. The field covered is roughly $65 \times 75$ arcsec with an image scale of $0.05 \mathrm{arcsec} / \mathrm{pixel}$, and a diffraction-limited resolution of $0.15 \mathrm{arcsec}$ FWHM. This field represents roughly half of the survey area covered, but the majority of the stars detected. Considerable structure in the PSF can be seen due to the AO system, serving to illustrate that great care must be taken when identifying faint companions to bright stars. 


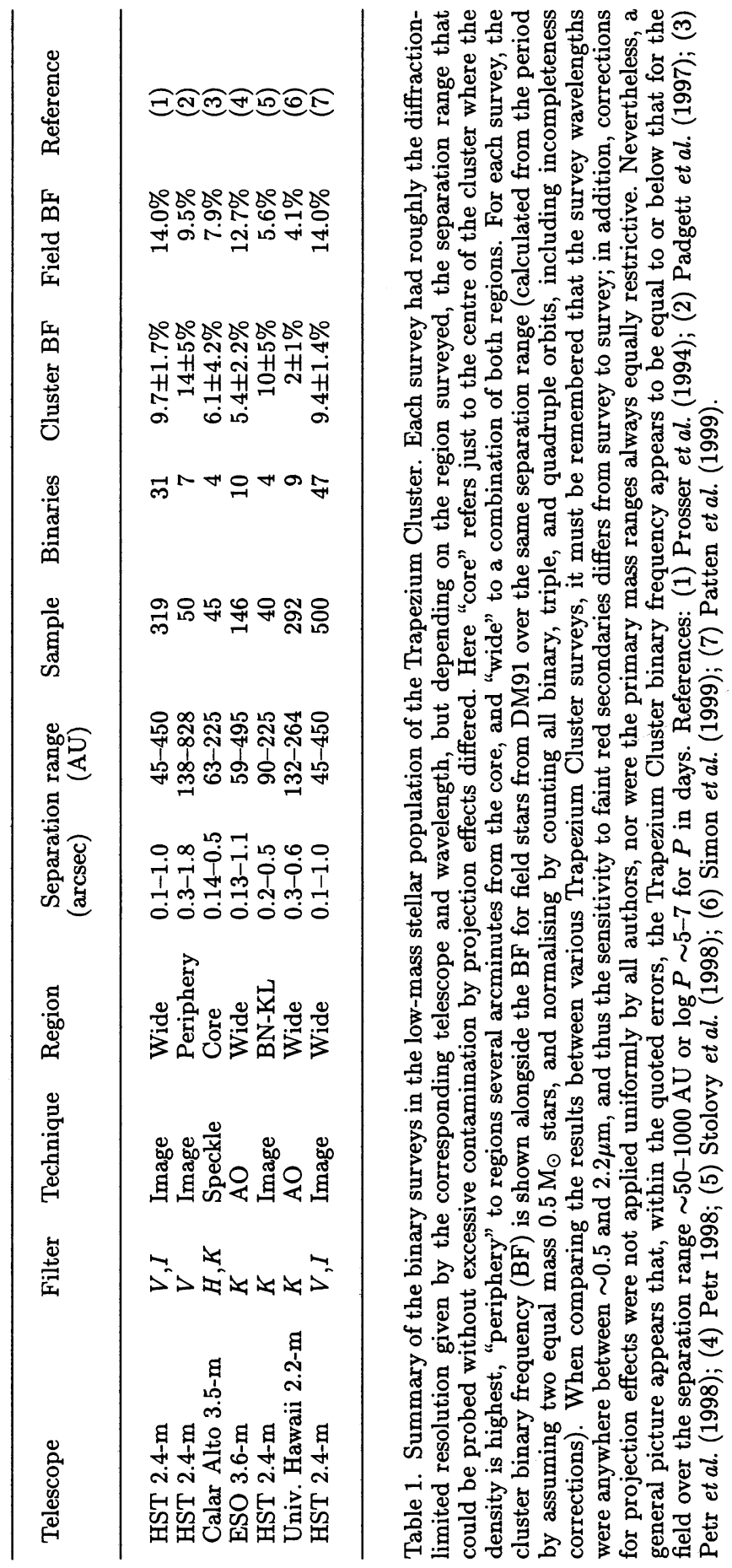


interactions would be less frequent and/or where stars ejected from binaries nearer the cluster core might be expected to lie. Similarly, the Stolovy et al. survey covers a region centred on the very young embedded BN-KL region, and primordial binaries may not yet have been disrupted. However, in both cases, it is worth re-emphasising that they are only $1 \sigma$ above the field. In contrast, the results of the Prosser etal. (1994), Petr etal. (1998), Petr (1998), Simon etal. (1999), and Patten etal. (1999) all lie below the field, and in all but the Petr et al. (1998) survey, they lie below the field by $2-3.5 \sigma$. Thus, if anything, the Trapezium Cluster binary frequency appears to be lower than that found in the field, and there is no evidence for the overabundance seen in Taurus-Auriga.

It would be premature to state definitively that the binary frequency of the Trapezium Cluster is lower than that of the field, given the differences in observational selection effects and completeness corrections that exist between the various surveys. Nevertheless, a tempting preliminary conclusion to draw is that some suitable admixture of regions like the Trapezium Cluster with its low binary frequency, and regions like Taurus-Auriga with its higher frequency, could be assembled to yield something like the field population. A very crude estimate suggests that this could be achieved by mixing one third Taurus-Auriga with two-thirds Trapezium Cluster. However, we should not forget that the surveys summarised thus far cover only a factor of twenty in binary separation and a corresponding two orders of magnitude in orbital period: by contrast, the DM91 field binaries cover eleven orders of magnitude. Therefore, it is clear that broader coverage is needed before such a wide-sweeping conclusion can be made.

\subsection{Extending to Wide Binaries}

Scally et al. (1999; SCM99) have taken the first steps in this direction, employing a novel technique to overcome the chance projection problem that makes it impossible to search for wide binaries in the cluster through imaging alone. By searching for common proper motion pairs in the optical proper motion study of Jones \& Walker (1988; JW88), they have been able to probe the separation range $\sim 1000-5000 \mathrm{AU}$. This paper is worth discussing in some detail here both for the interesting technique and because it yields an important new result.

At wide enough separations, the orbital velocity of one star around its companion is very low compared to the motion of the pair through the cluster, and thus in principle the pair should stand out in velocity space regardless of how confused the cluster is in spatial projection. SCM99 found three such common proper motion pairs within the separation range 1000-5000 AU in the JW88 data, but had then to assess how many of these could be due to chance alignments in velocity space and/or due to measurement uncertainties in the proper motions. SCM99 took a statistical approach to this, making model clusters using the Trapezium Cluster parameters (number of stars, core density, radial profile, velocity dispersion) as input to Monte Carlo simulations of the $x, y, z$ positions and $\dot{x}, \dot{y}, \dot{z}$ velocities for the stars. A distribution of errors matching that found by JW88 in their observations was imposed on the projected proper motions. Finally, a key component of the model clusters was the distribution of binary systems. The cluster was taken either to be binary-free or to follow the DM91 field star distribution. SCM99 then searched the artificial proper motion database for wide common proper motion binaries as they did for the actual 


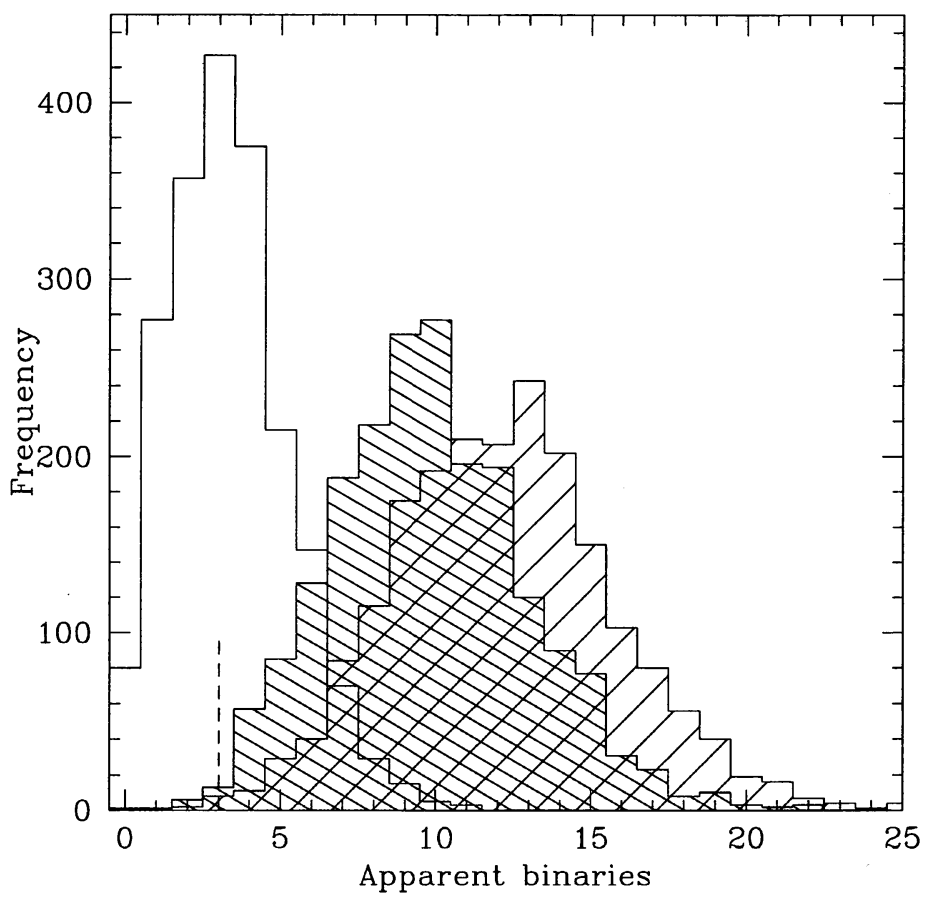

Figure 2. Simulations of the apparent frequency of wide common proper motion binaries in clusters from SCM99. In each case, 2000 model clusters with parameters similar to the Orion Nebula Cluster were created. Each was 'observed' with parameters appropriate to the real observations of JW88, counting the number of apparent wide common proper motion binaries. The empty histogram corresponds to clusters with no true binaries in the underlying model; the two hatched histograms correspond to model clusters with a binary population matching that found in the solar neighbourhood. The heavy-hatched histogram is for model clusters corrected for a brighter detection limit in the cluster centre than in the outer region; the light-hatched histogram is for clusters where the fainter detection limit is assumed throughout. The dashed line indicates the observational result of three apparent wide binaries found in the Orion Nebula Cluster, which corresponds well to the model with no true underlying binaries.

observational data. By repeating this procedure for many model clusters, they were then able to ascertain the mean number of wide common proper motion binaries that would be detected in both the binary-free and DM91 scenarios, and the standard deviations on those numbers.

The result is shown in Figure 2. The models that are binary-free nevertheless predict that three common proper motion pairs will appear through chance alignments in velocity space, i.e., exactly the number found in the JW88 data. On the other hand, a model with a DM91 distribution predicts that ten common proper motion pairs should be found, with just three being excluded at the $2.3 \sigma$ 
confidence level. Thus, although only marginally significant, the SCM99 result suggests that the Trapezium Cluster is deficient in wide binaries with respect to the field. Why this may be so is discussed in the next section. Regardless of the mechanism however, it would appear as though the wide field binaries must come from somewhere else. However, simply adding a Taurus-Auriga-like distribution (with its wide binaries) to a Trapezium Cluster-like one (with none apparently) in some linear mixture would not necessarily work, since filling in the wide binaries would once again raise the medium- to small-separation binary fraction relative to the field. This alludes to the important process of inverse population synthesis, in which the parameters of the present-day field binary population are used to deduce the fractions of stars that are born in the various kinds of star-forming regions. A more detailed discussion of this issue is beyond the scope of the present paper however.

\section{Theoretical Considerations}

This paper is primarily a review of observational studies of the binarity in the Trapezium Cluster, but it is nevertheless useful to glance at the relevant theoretical studies, most of which are discussed in detail elsewhere in these proceedings. The core problem is to understand why the present-day binary frequency of the Trapezium Cluster is lower than that of Taurus-Auriga. Essentially there are two possibilities: either fewer binaries formed to begin with in the Trapezium, or an initially high binary fraction was reduced to its present-day field-like value through some dynamical process.

The case for a lower binary fraction at formation was made by Durisen \& Sterzik (1994), who noted that models of binary formation predict that the number of binaries formed in a molecular cloud is inversely correlated with the cloud temperature. Since GMCs generally have core temperatures of $15-40 \mathrm{~K}$, as opposed to the $10-15 \mathrm{~K}$ found in low-mass dark clouds, they argued that a lower binary frequency was indeed expected for stars born in clusters in GMCs. However, no definitive test has yet been made of this hypothesis. The other possibility has been studied in great detail by Kroupa (see his paper in these proceedings and references therein), namely that interactions between stars in a dense cluster will destroy a substantial fraction of the primordial binary systems. Detailed N-body simulations of clusters have revealed the important effects that interactions can have on the period, eccentricity, and mass-ratio distributions, effects that space constraints preclude describing here.

However, a few words can be said on the binary frequency, which is most readily measured in the surveys described earlier in this paper. In particular, it is worth looking at the N-body calculations specifically tailored to simulating the Trapezium Cluster by Kroupa, Petr, \& McCaughrean (1999). The more successful Trapezium Cluster N-body models start either in virial equilibrium or in expansion. In these cases, there is a very high central density $\left(\sim 10^{6}\right.$ stars $\mathrm{pc}^{-3}$ ) initially, such that subsequent expansion of the cluster (due either to three and four-body interactions or sudden expulsion of gas as OB stars turn on, respectively) lowers the density to that observed $\left(\sim 5 \times 10^{4}\right.$ stars $\left.^{-3} c^{-3}\right)$ after $\sim 1$ Myr. This implies that many of the wider primordial binary systems, with separations greater than the typical intersystem separation of $\sim 2000 \mathrm{AU}$, overlap 
each other at the start of the simulation and are thus immediately disrupted before any dynamical evolution takes place. This effect instantaneously lowers the binary frequency by roughly $20 \%$ in the models, and yet in reality, such binaries would never have formed in the first place if the parent cloud cores were that closely packed together. This also suggests that if the cluster was indeed initially that dense, the lack of wide (1000-5000 AU) binaries in the cluster may actually be due to the initial conditions, not subsequent dynamical evolution.

Of course, once the cluster is allowed to expand, destructive encounters will affect systems, predominantly the wide binaries which have both a large cross-section for interaction and a low orbital binding energy compared to the average specific kinetic energy in the cluster. The hard/soft binary limit (i.e., the boundary below which binaries are relatively hard to disrupt) is a function of the cluster velocity dispersion, which changes as the cluster evolves. However, it is worth noting that for the present-day Trapezium Cluster with its 3D velocity dispersion of $\sim 4.5 \mathrm{~km} \mathrm{~s}^{-1}$ (JW88), the hard/soft binary limit lies at $\sim 45 \mathrm{AU}$ for a $1 \mathrm{M}_{\odot}$ system mass, i.e., right at the resolution limit of most extant surveys.

\section{Summary and Outlook}

Direct imaging surveys show that the binary frequency of young low-mass stars in the Orion Trapezium Cluster is broadly consistent with (or just below) that seen for field population over the separation range $\sim 50-1000$ AU. However, a statistical analysis of proper motion data shows a deficit of wider binaries with separations of $1000-5000 \mathrm{AU}$.

Theoretical studies show that an initially high, Taurus-Auriga-like binary frequency can have been reduced to the present-day, field-like frequency seen in the Trapezium Cluster. However, since alternate theories predict that they may never have formed, the non-existence of these wider visual binaries is a poor discriminant between the models. The acid test is to look at the binary population at small separations, small enough that they cannot have been destroyed via interactions within the cluster lifetime. If there are fewer of these short-period binaries than seen in Taurus-Auriga, then we will know that the birth environment has just as much an influence on the formation of binaries as on their subsequent evolution.

The present observational studies on 2-4-m telescopes have been limited to separations of $\sim 50 \mathrm{AU}$ or greater, at or above the present-day hard/soft binary limit, and by going to 8-10-m telescopes, we can improve this down to $\sim 20 \mathrm{AU}$. However, real breakthroughs will come with the application of interferometry, as then we will be able to probe below the equivalent hard/soft limit for the cluster when it was younger and denser. For example, getting to $10 \mathrm{AU}$ in Orion requires a $22-\mathrm{m}$ telescope at $2.2 \mu \mathrm{m}$, which is just the maximum interferometric baseline of the Large Binocular Telescope. Combined with its wide-field imaging interferometric capabilities, the LBT should be a perfect instrument for this next step. Beyond that, the Keck and VLT interferometers will yield maximum resolutions of 2 and $1 \mathrm{AU}$ at $2.2 \mu \mathrm{m}$ respectively. Since the binary frequency for the DM91 field star sample is $\sim 10 \%$ for separations of 1-10 AU (i.e., $\log P \sim 2.6$ 4.1 for a $1 \mathrm{M}_{\odot}$ system) and in Taurus-Auriga roughly double that, a survey of $\gtrsim 100$ members of the Trapezium Cluster would be needed to establish which 
population it more closely resembles. Finally, related to the issue of the dynamical disruption of binaries, a detailed star-by-star proper-motion survey will be needed to map the dynamical state of the cluster: this should be possible using the two-beam interferometric astrometer PRIMA on the VLTI, and the stable high spatial resolution, wide-field imaging capability of the NGST.

Acknowledgments. I would like to thank the first authors of the papers I have been involved in on the Trapezium Cluster binary population: Charles Prosser, Monika Petr, Matthew Bate, Aylwyn Scally, Pavel Kroupa, and Brian Patten. I would also like to thank Cathie Clarke and John Stauffer for instigating several of these studies. Special appreciation is reserved for my friend Hans Zinnecker, for imposing upon me the uniquely stimulating challenge of writing an after banquet speech in less than forty minutes. This work is supported in part by the DLR (50 OR 0004) and the EC Research Training Network "The Formation and Evolution of Young Stellar Clusters" (RTN1-1999-00436).

\section{References}

Allen, L. E., \& Hillenbrand, L. A. 2001, in The Orion Complex Revisited, ed. M. J. McCaughrean, ASP Conf. Ser., in press

Bally, J., O'Dell, C. R., \& McCaughrean, M. J. 2000, AJ, 119, 2919

Brandl, B., Brandner, W., Eisenhauer, F., Moffat, A. F. J., Palla, F., \& Zinnecker, H. 1999, A\&A, 352, L69

Clarke, C. J., Bonnell, I. A., \& Hillenbrand, L. A., in Protostars \& Planets IV, ed. V. Mannings, A. P. Boss, \& S. S. Russell, (Tucson: Univ. Arizona Press), 151

Combes, F. 1991, ARA\&A, 29, 195

Duchêne, G. 1999, A\&A, 341, 547

Duquennoy, A., \& Mayor, M. 1994, A\&A, 248, 485

Durisen, R. H., \& Sterzik, M. F. 1994, A\&A, 286, 84

Ghez, A. M., Matthews, K., \& Neugebauer, G. 1993, AJ, 106, 2005

Gomez, M., Hartmann, L., Kenyon, S. J., \& Hewett, R. 1993, AJ, 105, 1927

Herbig, G. H., \& Terndrup, D. M. 1986, ApJ, 307, 609

Hillenbrand, L. A. 1997, AJ, 113, 1733

Hillenbrand, L. A., \& Carpenter, J. M. 2000, ApJ, 540, 236

Hillenbrand, L. A., \& Hartmann, L. 1998, ApJ, 492, 540

Jones, B. F., \& Herbig, G. H. 1979, AJ, 84, 1872

Jones, B. F., \& Walker, M. F. 1988, AJ, 95, 1755

Köhler, R., \& Leinert, C. 1998, A\&A, 331, 977

Kroupa, P., Petr, M. G., \& McCaughrean, M. J. 1999, New A, 4, 495

Lada, E. A., Evans, N. J., Depoy, D. L., \& Gatley, I. 1991, ApJ, 371, L171

Lada, E. A., Strom, K. M., \& Myers, P. C. 1993, in Protostars \& Planets III, ed. E. H. Levy \& J. I. Lunine, (Tucson: Univ. Arizona Press), 245

Launhardt, R., \& Lada, E. A. 2001, in The Orion Complex Revisited, ed. M. J. McCaughrean, ASP Conf. Ser., in press 
Leinert, C., Zinnecker, H., Weitzel, N., Christou, J., Ridgway, S. T., Jameson, R., Haas, M., \& Lenzen, R. 1993, A\&A, 278, 129

Luhman, K. L., Rieke, G. H., Young, E. T., Cotera, A. S., Chen, H., Rieke, M. J., Schneider, G., \& Thompson, R. I. 2000, ApJ, 540, 1016

Mathieu, R. D. 1994, ARA\&A, 32, 465

McCaughrean, M. J. 2001, in From Darkness to Light, ed. T. Montmerle \& P. André, ASP Conf. Ser., in press

McCaughrean, M. J., \& Stauffer, J. R. 1994, AJ, 108, 1382

McCaughrean, M. J., Zinnecker, H., Rayner, J. T., \& Stauffer, J. R. 1996, in Disks and Outflows Around Young Stars, ed. S. Beckwith et al. , (Berlin: Springer), 33

Meyer, M. R., Adams, F. C., Hillenbrand, L. A., Carpenter, J. M., \& Larson, R. B. 2000, in Protostars \& Planets IV, ed. V. Mannings, A. P. Boss, \& S. S. Russell, (Tucson: Univ. Arizona Press), 121

Meyer, M., \& Lada, E. A. 2001, in The Orion Complex Revisited, ed. M. J. McCaughrean, ASP Conf. Ser., in press

Padgett, D. L., Strom, S. E., \& Ghez, A. M. 1997, ApJ, 477, 705

Patten, B. M., Stauffer, J. R., Hartmann, L. W., Hillenbrand, L. A., McCaughrean, M. J., Jones, B. F., \& Ghez, A. M. 1999, BAAS, 195, \#47.09

Petr, M. G. 1998, PhD thesis, University of Heidelberg

Petr, M. G., Coudé du Foresto, V., Beckwith, S. V. W., Richichi, A., \& McCaughrean, M. J. 1998, ApJ, 500, 825

Petr, M. G., McCaughrean, M. J., \& Zinnecker, H. 2001, in prep

Preibisch, T., Balega, Y., Hoffmann, K.-H., Weigelt, G., \& Zinnecker, H. 1999, New A, 4, 531

Prosser, C. F., Stauffer, J. R., Hartmann, L., Soderblom, D. R., Jones, B. F., Werner, M. W., \& McCaughrean, M. J. 1994, ApJ, 421, 517

Reipurth, B., \& Zinnecker, H. 1993, A\&A, 278, 81

Richichi, A., Leinert, C., Jameson, R., \& Zinnecker, H. 1994, A\&A, 287, 145

Scally, A., Clarke, C. J., \& McCaughrean, M. J. 1999, MNRAS, 306, 253

Simon, M., Close, L. M., \& Beck, T. 1999, AJ, 117, 1375

Simon, M., Ghez, A. M., Leinert, C., Cassar, L., Chen, W. P., Howell, R. R., Jameson, R. F., Matthews, K., Neugebauer, G., \& Richichi, A. 1995, ApJ, 443, 625

Stolovy, S., Burton, M. G., Erickson, E. F., Kaufman, M. J., Chrysostomou, A., Young, E. T., Colgan, S. W. J., Axon, D. J., Thompson, R. I., Rieke, M. J., \& Schneider, G. 1998, ApJ, 492, L151

Tatematsu, K., \& Wilson, T. L. 2001, in The Orion Complex Revisited, ed. M. J. McCaughrean, ASP Conf. Ser., in press

Weigelt, G., Balega, Y., Preibisch, T., Schertl, D., Schöller, M., \& Zinnecker, H. 1999, A\&A, 347, L15

Zinnecker, H., McCaughrean, M. J., \& Wilking, B. A. 1993, in Protostars \& Planets III, ed. E. H. Levy \& J. I. Lunine, (Tucson: Univ. Arizona Press), 429 